\title{
La poursuite de la progression de la maladie de Lyme en Ontario, Canada en 2017
}

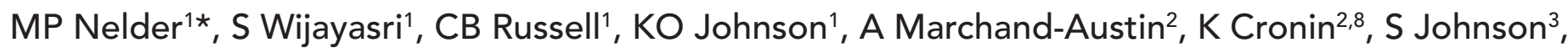 \\ T Badiani ${ }^{1}$, SN Patel ${ }^{4,5}$, D Sider ${ }^{6,7}$
}

\section{Résumé}

Contexte : La maladie de Lyme est une infection causée par le spirochète Borrelia burgdorferi et, dans la plus grande partie de l'Amérique du Nord, cette bactérie est transmise par la tique à pattes noires Ixodes scapularis. Les changements climatiques ont contribué à l'agrandissement de la distribution géographique des tiques à pattes noires en Ontario, ce qui a augmenté pour les Ontariens le risque de contracter la maladie de Lyme.

Objectif : Déterminer le nombre de cas et les taux d'incidence de même que la distribution géographique, saisonnière et démographique des cas de maladie de Lyme signalés en Ontario en 2017, comparativement aux tendances historiques.

Méthodologie : Les données des cas confirmés et probables de maladie de Lyme pour des épisodes étant survenus entre le $1^{\text {er }}$ janvier 2012 et le 31 décembre 2017 ont été extraites du Système intégré d'information sur la santé publique (SIISP). Elles portaient sur le bureau de santé publique (BSP) de résidence, la date de l'épisode, l'âge et le sexe. Les données populationnelles de Statistique Canada ont servi à calculer les taux d'incidence pour 100000 habitants à l'échelle des BSP et de la province. Le nombre de cas déclarés en 2017 par BSP de résidence selon le mois de l'épisode, l'âge et le sexe a été comparé à la moyenne des cinq dernières années au cours de la période 2012-2016.

Résultats : En 2017, 959 cas probables et confirmés de maladie de Lyme ont été signalés en Ontario. Ce nombre était trois fois plus élevé que la moyenne de 313 cas des cinq dernières années (de 2012 à 2016). Le taux d'incidence provincial pour 2017 était de 6,7 cas pour 100000 habitants, même s'il variait de façon marquée d'un BSP à l'autre. Les taux d'incidence les plus élevés ont été observés dans le district de Leeds, Grenville et Lanark (128,8 cas pour 100000 habitants), dans le district de Kingston, Frontenac et Lennox et Addington (87,2 cas pour 100000 habitants), dans les comtés de Hastings et de Prince Edward (28,6 cas pour 100000 habitants), à Ottawa (18,1 cas pour 100000 habitants) et dans l'est de I'Ontario (13,5 cas pour 100000 habitants). Les cas sont survenus surtout pendant les mois de juin à septembre et étaient plus fréquents chez les personnes de sexe masculin de 5 à 14 ans et de 50 à 69 ans.

Conclusion : En 2017, l'incidence de la maladie de Lyme a démontré une nette croissance en Ontario, tout particulièrement dans l'est de la province. Si les conditions météorologiques et les tendances climatiques actuelles se poursuivent, les tiques à pattes noires porteuses de pathogènes, comme ceux causant la maladie de Lyme, continueront à s'étendre dans les habitats propices. Surveiller l'étendue de leur expansion géographique guidera les futures actions cliniques et de santé publique afin de détecter et de prévenir les répercussions de la maladie de Lyme en Ontario.

Citation proposée : Nelder MP, Wijayasri S, Russell CB, Johnson KO, Marchand-Austin A, Cronin K, Johnson S, Badiani T, Patel SN, Sider D. La poursuite de la progression de la maladie de Lyme en Ontario, Canada en 2017. Relevé des maladies transmissibles au Canada 2018;44(10):262-8. https://doi.org/10.14745/ccdr.v44i10a01f
Affiliations

${ }^{1}$ Maladies entériques, zoonotiques et à transmission vectorielle, Maladies transmissibles, préparation aux situations d'urgence et intervention en cas d'urgence, Santé publique Ontario, Toronto (Ontario)

2 Surveillance en laboratoire et gestion des données, Laboratoires de Santé publique Ontario, Santé publique Ontario, Toronto (Ontario)

${ }^{3}$ Services de l'informatique, Services axés sur le savoir, Santé publique Ontario, Toronto (Ontario)

${ }^{4}$ Laboratoires de Santé publique Ontario - Toronto, Santé publique Ontario, Toronto (Ontario)

${ }^{5}$ Département de médecine de laboratoire et de pathobiologie, Université de Toronto, Toronto (Ontario)

${ }^{6}$ Maladies transmissibles, préparation aux situations d'urgence et intervention en cas d'urgence, Santé publique Ontario, Toronto (Ontario)

${ }^{7}$ Département d'épidémiologie clinique et de biostatistique, Université McMaster, Hamilton (Ontario)

${ }^{8}$ Laboratoire national de microbiologie, Agence de la santé publique du Canada, Winnipeg (Manitoba)

*Correspondance: mark.nelder@ oahpp.ca

Mots-clés : Borrelia, épidémiologie, expansion, tiques, Ixodes, santé publique, distribution géographique, risque, surveillance 


\section{Introduction}

La maladie de Lyme est une infection à spirochète causée par la bactérie Borrelia burgdorferi et qui, dans la majeure partie de l'Amérique du Nord, est transmise par la morsure d'une tique à pattes noires, Ixodes scapularis, infectée. L'infection à la bactérie $B$. burgdorferi commence typiquement par une éruption cutanée et des symptômes ressemblant à l'influenza (1-6). Dans la majorité des cas, le traitement à l'aide d'antibiotiques permet une guérison complète. Cependant, si elle n'est pas détectée et qu'on ne la traite pas, l'infection peut évoluer en maladie disséminée présentant une probabilité de morbidité accrue et des séquelles à long terme, le syndrome de la maladie de Lyme post-traitement (7-9).

Le nombre de cas de maladie de Lyme a augmenté au sextuple de 2009 à 2015 au Canada, la majorité des cas ayant été signalés en Ontario (10). Ce fait a été associé à la distribution géographique des tiques à pattes noires au Canada, y compris dans le nord de I'Ontario. Cette expansion est attribuable en grande partie aux changements climatiques, c.-à-d. à l'augmentation du nombre de degrés-jours annuels cumulatifs au-dessus de $0^{\circ} \mathrm{C}(11-13)$. Les responsables de la santé publique en Ontario surveillent les risques de maladie de Lyme en assurant la surveillance de la tique à pattes noires et en déclarant les cas de maladie de Lyme.

Une compréhension de l'épidémiologie de la maladie de Lyme est essentielle afin de guider les efforts cliniques et de santé publique dans le but d'augmenter la sensibilisation, la prévention, la détection précoce et l'atténuation. L'objectif de la présente étude était de déterminer le nombre de cas et les taux d'incidence de même que la distribution géographique, saisonnière et démographique des cas de maladie de Lyme signalés en Ontario en 2017 et de les comparer aux tendances historiques des cinq dernières années.

\section{Méthodologie}

\section{Sites et population de l'étude}

L'Ontario compte environ 14,2 millions de personnes, largement concentrées dans le sud de la province (14). Dans le Sud de I'Ontario, qui jouit d'un climat continental humide modéré, on trouve un mélange de territoires agricoles, de forêts de feuillus et mixtes, et d'environnements bâtis (15). En 2017, 36 bureaux de santé publique (BSP) administraient les services de santé publique en Ontario, y compris le suivi des cas de maladie de Lyme chez les humains et la surveillance des tiques à pattes noires.

\section{Collecte et analyse des données}

Lorsqu'ils sont informés d'un nouveau cas de maladie de Lyme, les professionnels de la santé publique procédient au suivi des cas et collectient des renseignements concernant la démographie, l'exposition, les symptômes de même que les cas d'hospitalisation et de décès. Si le cas correspondait à la définition provinciale d'un cas de surveillance (voir l'encadré), l'ensemble des données est alors transmis au Système intégré d'information sur la santé publique (SIISP) provincial.

Définitions de cas de surveillance par l'Ontario pour des cas confirmés et probables de maladie de Lyme (de 2012 à 2017) : Ontario, Canada (16)

\section{CAS CONFIRMÉ}

- cas d'érythème chronique migrateur d'un diamètre supérieur à $5 \mathrm{~cm}$ confirmé par un clinicien chez une personne ayant déjà habité ou visité une région où la maladie de Lyme est endémique ou qui est une région à risque $\mathrm{OU}$

- $\quad$ preuve clinique de maladie de Lyme confirmée en laboratoire par amplification en chaîne par polymérase (polymerase chain reaction [PCR]) ou par culture OU

- $\quad$ preuve clinique de maladie de Lyme soutenue par des méthodes sérologiques en laboratoire chez une personne ayant déjà habité ou visité une région où la maladie de Lyme est endémique ou qui est une région à risque

\section{CAS PROBABLE}

- $\quad$ preuve clinique de maladie de Lyme soutenue par des méthodes sérologiques en laboratoire chez une personne n'ayant jamais habité ni visité une région où la maladie de Lyme est endémique ou qui est une région à risque $\mathrm{OU}$

- cas d'érythème chronique migrateur d'un diamètre supérieur à $5 \mathrm{~cm}$ confirmé par un clinicien chez une personne n'ayant jamais habité ni visité une région où la maladie de Lyme est endémique ou qui est une région à risque

Dans le cadre de la présente étude, nous avons extrait les données du SIISP pour des cas confirmés et probables de maladie de Lyme concernant des épisodes s'étant manifestés entre le $1^{\text {er }}$ janvier 2012 et le 31 décembre 2017 et nous avons calculé le nombre et la proportion de cas par BSP de résidence, selon le mois de l'épisode, l'âge et le sexe.

Pour une personne donnée, nous avons utilisé son BSP de résidence et la date de l'épisode le plus ancien dans le but de calculer le nombre de cas et les taux d'incidence. Les dates des épisodes nous ont aussi permis de déterminer le caractère saisonnier. La date d'un épisode a été définie comme la date de l'apparition des premiers symptômes, celle de la collecte du spécimen ou celle où le problème a été signalé. En raison du caractère incomplet des données et de la possibilité d'occasions d'exposition multiples, nous n'avons pas essayé de déterminer si un cas donné avait été exposé localement (exposition à I'intérieur des limites du BSP de résidence) ou au cours d'un déplacement (à l'extérieur des limites du BSP de résidence). 
Les estimations (de 2012 à 2016) et les projections (2017) de population, obtenues de Statistique Canada au moyen de SavoirSanté Ontario, ont servi à calculer les taux d'incidence pour 100000 habitants pour la province et le BSP. Nous avons regroupé les taux d'incidence de la maladie de Lyme par BSP afin de les cartographier à l'aide du système d'information géographique ESRI ArcGIS v. 10.3 (Environmental Systems Research Institute, Inc., Redlands, Californie, États-Unis [É.-U.]). Les taux d'incidence par BSP ont ensuite été divisés en catégories de façon manuelle : 0 , de 0,1 à 5 , de 5,1 à 10 , de 10,1 à 30 et taux supérieurs à 30. Les données de 2017 ont été comparées à la moyenne des cinq dernières années au cours de la période de 2012 à 2016. Des analyses descriptives ont été réalisées à l'aide de Microsoft Excel 2010 (Microsoft, Redmond, Washington, É.-U.) et SAS 9.3 (Statistical Analysis System, Cary, Caroline du Nord, É.-U.).

Le présent article porte sur des activités de surveillance de routine et non pas sur un projet de recherche, de telle sorte que I'approbation par un comité d'éthique n'était pas requise. Les données sont disponibles sur demande auprès de Santé publique Ontario (SPO) à l'adresse https://www.publichealthontario.ca/fr/ Qui-sommes-nous/Pages/privacy.aspx.

\section{Résultats}

\section{Dénombrement des cas, taux d'incidence et distribution géographique}

En 2017, 959 cas confirmés et probables de maladie de Lyme ont été déclarés en Ontario (figure 1).

Figure 1: Nombre de cas probables et confirmés et taux d'incidence pour 100000 habitants de maladie de Lyme par année : Ontario, Canada (de 2012 à 2017)

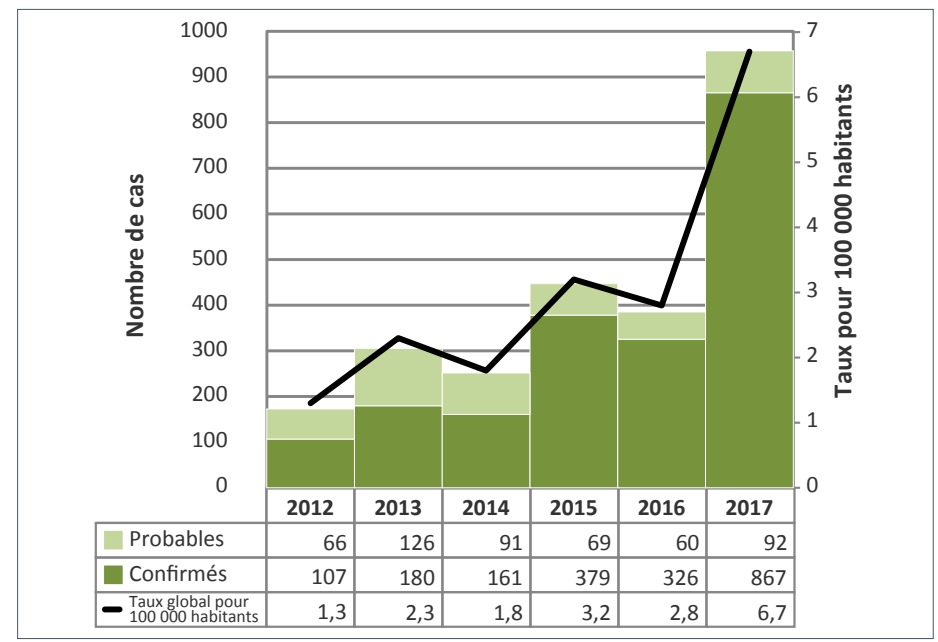

En 2017, le total était 3,1 fois plus élevé que la moyenne de 313 cas des cinq dernières années (de 2012 à 2016) (tableau 1). La majorité des cas signalés en 2017 l'ont été dans le district de Leeds, Grenville et Lanark (LGL) ( $n=219,22,8 \%$ du total provincial), Kingston, Frontenac et Lennox et Addington (KFL) ( $n=180,18,8 \%$ ) et Ottawa ( $n=180,18,8 \%$ ). Ces trois BSP représentaient $9,7 \%$ de la population de l'Ontario, mais c'est eux qui ont déclaré $60,4 \%$ des cas de maladie de Lyme de la province. En 2017, le nombre de cas du district de LGL était 4,6 fois supérieur à la moyenne des cinq dernières années; le nombre de cas de KFL et d'Ottawa étaient respectivement 3,6 fois et 3,8 fois plus élevés que la moyenne des cinq dernières années.

Tableau 1 : Nombre de cas probables et confirmés et taux d'incidence de maladie de Lyme par bureau de santé publique : Ontario, Canada (2017)

\begin{tabular}{|c|c|c|c|c|}
\hline $\begin{array}{c}\text { Bureau de santé } \\
\text { publique }\end{array}$ & $\begin{array}{l}\text { Nombres } \\
\text { de cas }\end{array}$ & $\%$ & $\begin{array}{l}\text { Moyenne des } \\
\text { cinq dernières } \\
\text { années }\end{array}$ & $\begin{array}{l}\text { Incidence } \\
\text { pour } \\
100000 \\
\text { habitants }\end{array}$ \\
\hline $\begin{array}{l}\text { District d'Algoma } \\
\text { (ALG) }\end{array}$ & 2 & 0,2 & 2,5 & 1,7 \\
\hline $\begin{array}{l}\text { Comté de Brant } \\
\text { (BRN) }\end{array}$ & 1 & 0,1 & 2,0 & 0,7 \\
\hline Chatham-Kent (CHK) & 3 & 0,3 & 2,2 & 2,9 \\
\hline $\begin{array}{l}\text { Ville de Hamilton } \\
\text { (HAM) }\end{array}$ & 5 & 0,5 & 6,2 & 0,9 \\
\hline Ottawa (OTT) & 180 & 18,8 & 47,4 & 18,1 \\
\hline $\begin{array}{l}\text { Région de Durham } \\
\text { (DUR) }\end{array}$ & 46 & 4,8 & 14,2 & 6,7 \\
\hline $\begin{array}{l}\text { Est de l'Ontario } \\
(\text { EOH) }\end{array}$ & 28 & 2,9 & 17,0 & 13,5 \\
\hline $\begin{array}{l}\text { Elgin-St. Thomas } \\
\text { (ELG) }\end{array}$ & 0 & 0,0 & 1,3 & 0,0 \\
\hline Grey Bruce (GBO) & 3 & 0,3 & 2,0 & 1,8 \\
\hline $\begin{array}{l}\text { Haldimand-Norfolk } \\
\text { (HDN) }\end{array}$ & 7 & 0,7 & 2,2 & 6,3 \\
\hline $\begin{array}{l}\text { District de } \\
\text { Haliburton-Kawartha- } \\
\text { Pine Ridge (HKP) } \\
\end{array}$ & 17 & 1,8 & 5,2 & 9,3 \\
\hline $\begin{array}{l}\text { Région de Halton } \\
\text { (HAL) }\end{array}$ & 13 & 1,4 & 7,4 & 2,2 \\
\hline $\begin{array}{l}\text { Comtés de Hastings } \\
\text { et de Prince Edward } \\
\text { (HPE) }\end{array}$ & 47 & 4,9 & 18,2 & 28,6 \\
\hline $\begin{array}{l}\text { Comté de Huron } \\
\text { (HUR) }\end{array}$ & 1 & 0,1 & 1,7 & 1,7 \\
\hline $\begin{array}{l}\text { Kingston, Frontenac } \\
\text { et Lennox et } \\
\text { Addington (KFL) }\end{array}$ & 180 & 18,8 & 49,6 & 87,2 \\
\hline $\begin{array}{l}\text { Comté de Lambton } \\
\text { (LAM) }\end{array}$ & 1 & 0,1 & 3,0 & 0,8 \\
\hline $\begin{array}{l}\text { District de Leeds, } \\
\text { Grenville et Lanark } \\
\text { (LGL) }\end{array}$ & 219 & 22,8 & 47,8 & 128,8 \\
\hline $\begin{array}{l}\text { Middlesex-London } \\
\text { (MSL) }\end{array}$ & 15 & 1,6 & 5,2 & 3,1 \\
\hline $\begin{array}{l}\text { Région de Niagara } \\
\text { (NIA) }\end{array}$ & 19 & 2,0 & 9,2 & 4,1 \\
\hline $\begin{array}{l}\text { District de North Bay } \\
\text { Parry Sound (NPS) } \\
\end{array}$ & 1 & 0,1 & 1,3 & 0,8 \\
\hline Nord-Ouest (NWR) & 2 & 0,2 & 3,2 & 2,5 \\
\hline $\begin{array}{l}\text { Comté d'Oxford } \\
\text { (OXF) }\end{array}$ & 2 & 0,2 & 1,8 & 1,8 \\
\hline
\end{tabular}


Tableau 1 : (suite) Nombre de cas probables et confirmés et taux d'incidence de maladie de Lyme par bureau de santé publique : Ontario, Canada (2017)

\begin{tabular}{|c|c|c|c|c|}
\hline $\begin{array}{c}\text { Bureau de santé } \\
\text { publique }^{a}\end{array}$ & $\begin{array}{l}\text { Nombres } \\
\text { de cas }\end{array}$ & $\%$ & $\begin{array}{l}\text { Moyenne des } \\
\text { cinq dernières } \\
\text { années }\end{array}$ & $\begin{array}{c}\text { Incidence } \\
\text { pour } \\
100000 \\
\text { habitants }\end{array}$ \\
\hline Région de Peel (PEE) & 12 & 1,3 & 5,8 & 0,8 \\
\hline $\begin{array}{l}\begin{array}{l}\text { District de Perth } \\
(\mathrm{PDH})\end{array} \\
\end{array}$ & 1 & 0,1 & 1,3 & 1,3 \\
\hline \begin{tabular}{|l} 
Comté et ville de \\
Peterborough (PTC)
\end{tabular} & 12 & 1,3 & 2,8 & 8,4 \\
\hline Porcupine (PQP) & 0 & 0,0 & 1,0 & 0,0 \\
\hline $\begin{array}{l}\text { Comté de Renfrew et } \\
\text { district (REN) }\end{array}$ & 7 & 0,7 & 3,2 & 6,5 \\
\hline \begin{tabular}{|l|} 
District de Simcoe \\
Muskoka (SMD) \\
\end{tabular} & 12 & 1,3 & 3,8 & 2,1 \\
\hline $\begin{array}{l}\text { District de Sudbury } \\
\text { (SUD) }\end{array}$ & 3 & 0,3 & 1,5 & 1,5 \\
\hline $\begin{array}{l}\text { District de Thunder } \\
\text { Bay (THB) }\end{array}$ & 0 & 0,0 & 1,0 & 0,0 \\
\hline Timiskaming (TSK) & 0 & 0,0 & 0,0 & 0,0 \\
\hline Toronto (TOR) & 76 & 8,0 & 31,0 & 2,6 \\
\hline Waterloo (WAT) & 7 & 0,7 & 4,2 & 1,3 \\
\hline $\begin{array}{l}\text { Wellington-Dufferin- } \\
\text { Guelph (WDG) }\end{array}$ & 9 & 0,9 & 2,4 & 3,1 \\
\hline $\begin{array}{l}\text { Windsor-comté } \\
\text { d'Essex (WEC) }\end{array}$ & 7 & 0,7 & 4,0 & 1,7 \\
\hline Région de York (YRK) & 21 & 2,2 & 9,8 & 1,8 \\
\hline Total & 959 & $100^{b}$ & $\begin{array}{r}313 \\
\text { (moyenne) }\end{array}$ & $\begin{array}{r}6,7 \\
\text { (moyenne) }\end{array}$ \\
\hline
\end{tabular}

Abréviation de trois lettres

'La somme des pourcentages ne donne pas $100 \%$ en raison de l'arrondissement des valeurs

Les taux d'incidence les plus élevés en 2017 se situaient dans les districts de LGL (128,8 cas pour 100000 habitants), KFL (87,2 cas pour 100000 habitants), HPE (28,6 cas pour 100000 habitants), à Ottawa (18,1 cas pour 100000 habitants) et dans l'est de I'Ontario (13,5 cas pour 100000 habitants) (figure 2).

Figure 2 : Taux d'incidence de la maladie de Lyme (pour 100000 habitants) par bureau de santé publique :

Ontario, Canada (2017)

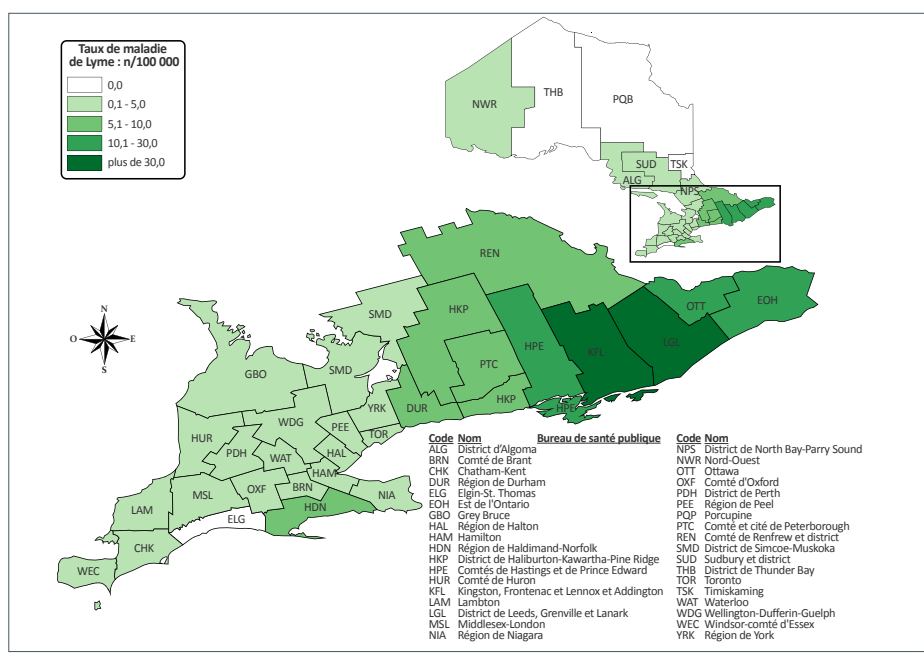

Abréviation : $n$, nombre

\section{Caractère saisonnier}

En 2017, la majorité des cas sont survenus entre les mois de juin et septembre; juillet présentant le plus grand nombre de cas $(n=369)$ (figure 3$)$. Le nombre de cas mensuel dépassait la moyenne des cinq dernières années pour les mois de juin ( 2,7 fois plus élevé), juillet ( 3,8 fois), août ( 4,7 fois) et septembre (3 fois).

Figure 3 : Nombre de cas probables et confirmés de maladie de Lyme selon le mois de l'épisode en 2017, par rapport à la moyenne des cinq dernières années (de 2012 à 2016) : Ontario, Canada

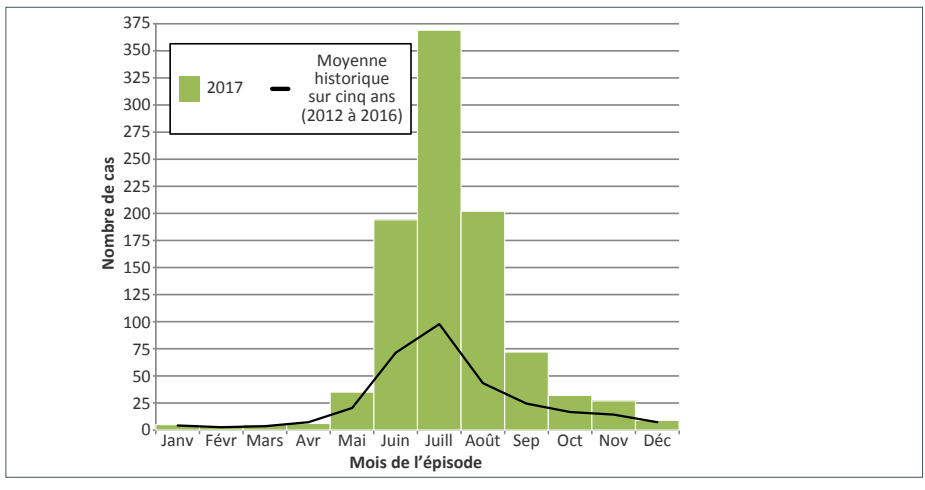

\section{Âge et sexe}

Le nombre de cas de maladie de Lyme en Ontario suivait un comportement bimodal en 2017, avec un nombre de cas relativement plus élevé pour les groupes d'âges de 5 à 14 ans et de 50 à 69 ans et relativement plus faible pour les jeunes de 15 à 24 ans (figure 4). Les personnes de sexe masculin-féminin représentaient plus de la moitié des cas $-59,8 \%$. Le ratio entre

Figure 4 : Nombre de cas confirmés et probables de maladie de Lyme selon le groupe d'âge et le sexe en 2017, comparativement à la moyenne des cinq dernières années (de 2012 à 2016): Ontario, Canada ${ }^{a}$

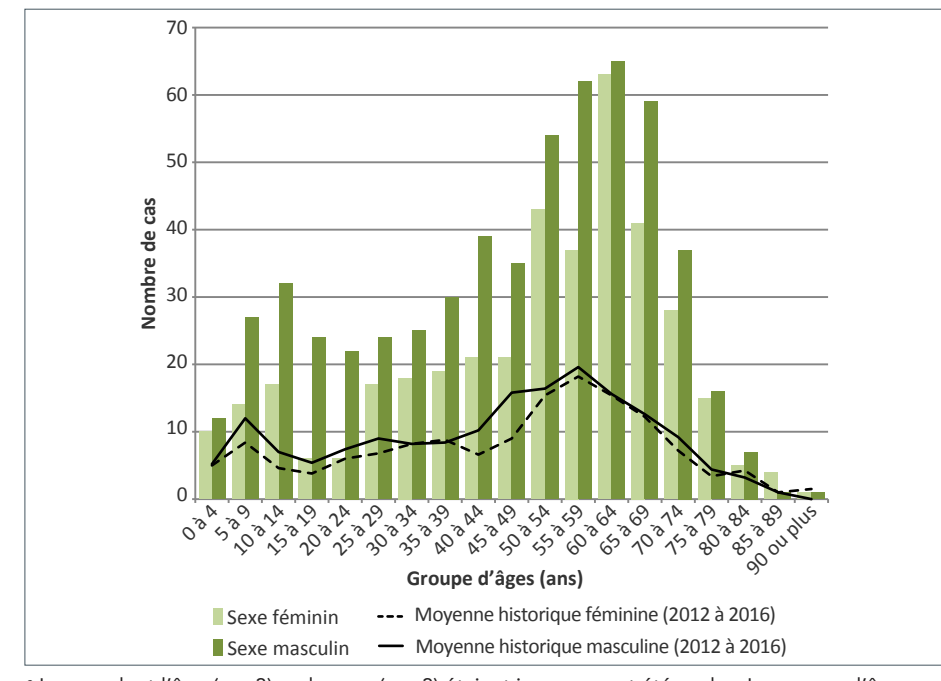

a Les cas dont l'âge $(n=3)$ ou le sexe $(n=3)$ étaient inconnus ont été exclus. Le groupe d'âges fait référence au groupe d'âges de la personne au moment de sa maladies 
les personnes de masculin et féminin était plus élevé que celui de la moyenne des cinq dernières années pour la plupart des groupes d'âges. Il était presque deux fois plus élevé dans les groupes d'âges de 5 à 14 ans, de 35 à 39 ans, de 40 à 49 ans et de 55 à 59 ans et était de trois à quatre fois plus élevé pour les groupes d'âges de 15 à 19 ans et de 20 à 24 ans.

\section{Discussion}

L'incidence de la maladie de Lyme en 2017, en Ontario, était à son plus haut niveau enregistré depuis qu'elle est devenue une maladie à déclaration obligatoire en 1998. L'augmentation de son incidence en Ontario n'est pas uniforme, mais elle est concentrée dans la partie est de la province qui abrite davantage de tiques à pattes noires et un pourcentage relativement plus élevé $(17,18 \%)$ de tiques à pattes noires porteuses de la bactérie B. burgdorferi. Comme dans le reste du Canada et ailleurs, les cas de maladie de Lyme étaient plus fréquents entre les mois de juin et de septembre, et ils étaient plus nombreux chez les personnes de 5 à 14 ans et de 50 à 69 ans de même que chez les personnes de sexe masculin.

La force de notre étude est de présenter les données disponibles les plus à jour sur l'incidence de la maladie de Lyme en Ontario, de même qu'une analyse des tendances géographiques, saisonnières et démographiques de cette infection.

Plusieurs limites doivent être prises en considération. Premièrement, la véritable incidence d'une infection comme la maladie de Lyme souffre de différents degrés de sous-déclaration en raison d'un éventail de facteurs, comme un niveau variable de sensibilisation à la maladie, de comportements de consultation pour des soins de santé, de diagnostics cliniques, de comportements en lien avec la déclaration ainsi que de traitement clinique de cas de maladie de Lyme localisés de façon précoce sans être signalés aux $\operatorname{BSP}(19,20)$. Bien que le degré de sous-déclaration à l'échelle provinciale n'ait pas été déterminé, nous supposons que le nombre de cas observés par la surveillance passive ressemble au nombre de cas confirmés par sérologie.

Deuxièmement, le SIISP est un système de déclaration des maladies qui permet la mise à jour continue des données saisies précédemment. II en résulte que les données extraites de ce système représentent un portrait instantané de la base de données au moment de l'extraction et qu'elles peuvent changer par rapport aux déclarations précédentes ou subséquentes. Troisièmement, comme il n'était pas possible d'évaluer si les infections à la bactérie $B$. burgdorferi étaient acquises localement ou lors de déplacements à l'extérieur, le BSP de résidence ne correspond pas nécessairement au lieu de l'exposition. Néanmoins, pour un cas donné, le site le plus vraisemblable pour une exposition est près de la maison ou, sur un territoire plus vaste, dans le secteur de son BSP de résidence $(21,22)$.

\section{Prochaines étapes}

Ces données ont des répercussions sur la recherche comme sur la santé publique. Les banques de données de maladies à déclaration obligatoire, comme le SIISP, permettent d'explorer l'épidémiologie de la maladie de Lyme en Ontario. Par exemple, des projets de recherche faisant appel au SIISP et à d'autres bases de données relatives aux soins de santé pourraient examiner les tendances spatio-temporelles concernant la déclaration et le traitement de cas pour lesquels une confirmation en laboratoire est présente ou non.

Ces données ont des répercussions importantes sur la santé publique en ce sens que les tendances observées offrent l'occasion de mieux cibler la sensibilisation à la prévention de la maladie de Lyme, comme des programmes éducatifs pour les enfants et les parents, et la nécessité de se protéger lors des activités estivales. Chez la tique, l'été est associé au stade de la nymphe. Une sensibilisation accrue à la petitesse de sa taille à cette période pourrait aussi être utile.

\section{Conclusion}

L'incidence de la maladie de Lyme est en croissance en Ontario. Cette tendance va probablement se poursuivre en raison de la progression des changements climatiques qui permettent aux tiques à pattes noires de survivre et de se propager dans de nouvelles régions. La surveillance continue des cas chez l'humain et de la distribution géographique des tiques peut continuer à guider les interventions cliniques et de santé publique afin de prévenir, de détecter et d'atténuer les séquelles de la maladie de Lyme en Ontario.

\section{Déclaration des auteurs}

M. P. N. - Conceptualisation, méthodologie, analyse, interprétation, rédaction de la première ébauche, révision et édition

C. B. R., D. S., K. O. J., S. W. - Conceptualisation, méthodologie, analyse, interprétation, rédaction de la première ébauche (en partie), révision et édition

A.-M. A., K. C., S. J., S. N. P., T. B. - Méthodologie, analyse, interprétation, révision et édition

\section{Conflit d'intérêts}

Aucun.

\section{Remerciements}

Nous tenons à remercier nos collègues de la santé publique partout en Ontario pour leur engagement continu envers la gestion de cas, la surveillance et la déclaration de la maladie de Lyme. Nous remercions Lisa Fortuna (Santé publique Ontario), Shelley Deeks (Santé publique Ontario), Patricia Huston (Agence de la santé publique du Canada) et un réviseur anonyme pour la révision des versions antérieures de ce texte et pour avoir contribué à son amélioration. 
https://doi.org/10.1086/318082. PubMed (https://www.ncbi. $\mathrm{nlm}$.nih.gov/entrez/query.fcgi?cmd=Retrieve\&db=PubMed\&l ist_uids $=11133377 \&$ dopt $=$ Abstract)

Santé publique Ontario a soutenu cette étude dans le cadre de son mandat principal.

\section{Références}

1. Arvikar SL, Steere AC. Diagnosis and treatment of Lyme arthritis. Infect Dis Clin North Am 2015 Jun;29(2):269-80. https://doi.org/10.1016/j.idc.2015.02.004. PubMed (https:// www.ncbi.nlm.nih.gov/entrez/query.fcgi?cmd=Retrieve\&db= PubMed\&list_uids $=25999223 \&$ dopt $=$ Abstract )

2. Smith RP, Schoen RT, Rahn DW, Sikand VK, Nowakowski J, Parenti DL, Holman MS, Persing DH, Steere AC. Clinical characteristics and treatment outcome of early Lyme disease in patients with microbiologically confirmed erythema migrans. Ann Intern Med 2002 Mar;136(6):421-8. https://doi. org/10.7326/0003-4819-136-6-200203190-00005. PubMed (https://www.ncbi.nlm.nih.gov/entrez/query.fcgi?cmd=Retrie ve\&db=PubMed\&list_uids=11900494\&dopt=Abstract)

3. Halperin JJ. Nervous system Lyme disease. Clin Lab Med 2015 Dec;35(4):779-95. https://doi.org/10.1016/j. cll.2015.07.002. PubMed (https://www.ncbi.nlm.nih. gov/entrez/query.fcgi?cmd=Retrieve\&db=PubMed\&lis t_uids=26593257\&dopt=Abstract)

4. Robinson ML, Kobayashi T, Higgins Y, Calkins H, Melia MT. Lyme carditis. Infect Dis Clin North Am 2015 Jun;29(2):25568. https://doi.org/10.1016/j.idc.2015.02.003. PubMed (https://www.ncbi.nlm.nih.gov/entrez/query.fcgi?cmd=Retrie ve\&db=PubMed\&list_uids=25999222\&dopt=Abstract)

5. Nadelman RB. Erythema migrans. Infect Dis Clin North Am 2015 Jun;29(2):211-39. https://doi.org/10.1016/j. idc.2015.02.001. PubMed (https://www.ncbi.nlm.nih. gov/entrez/query.fcgi?cmd=Retrieve\&db=PubMed\&lis t_uids=25999220\&dopt=Abstract)

6. Steere AC, Bartenhagen NH, Craft JE, Hutchinson GJ, Newman JH, Rahn DW, Sigal LH, Spieler PN, Stenn KS, Malawista SE. The early clinical manifestations of Lyme disease. Ann Intern Med 1983;99(1):76-82. https://doi. org/10.7326/0003-4819-99-1-76

7. Aucott JN. Posttreatment Lyme disease syndrome. Infect Dis Clin North Am 2015 Jun;29(2):309-23. https://doi. org/10.1016/j.idc.2015.02.012. PubMed (https://www.ncbi. $\mathrm{n} / \mathrm{m}$.nih.gov/entrez/query.fcgi? $\mathrm{cmd}=$ Retrieve\&db=PubMed\&l ist_uids=25999226\&dopt=Abstract)

8. Kalish RA, Kaplan RF, Taylor E, Jones-Woodward L, Workman $\mathrm{K}$, Steere AC. Evaluation of study patients with Lyme disease, 10-20-year follow-up. J Infect Dis 2001 Feb;183(3):453-60.
9. Shadick NA, Phillips CB, Logigian EL, Steere AC, Kaplan RF, Berardi VP, Duray PH, Larson MG, Wright EA, Ginsburg $\mathrm{KS}$, Katz JN, Liang MH. The long-term clinical outcomes of Lyme disease. A population-based retrospective cohort study. Ann Intern Med 1994 Oct;121(8):560-7. https://doi. org/10.7326/0003-4819-121-8-199410150-00002. PubMed (https://www.ncbi.nlm.nih.gov/entrez/query.fcgi?cmd=Retrie ve\&db=PubMed\&list_uids=8085687\&dopt=Abstract)

10. Gasmi S, Ogden NH, Lindsay LR, Burns S, Fleming S, Badcock J, Hanan S, Gaulin C, Leblanc MA, Russell C, Nelder M, Hobbs L, Graham-Derham S, Lachance L, Scott AN, Galanis E, Koffi JK. Surveillance de la Lyme au Canada, de 2009 à 2015. Relevé des maladies transmissibles au Canada. 2017;43(10):219-25. https://doi.org/10.14745/ccdr. v43i10a01f

11. Clow KM, Leighton PA, Ogden NH, Lindsay LR, Michel P, Pearl, Jardine CM. Northward range expansion of Ixodes scapularis evident over a short timescale in Ontario, Canada. PLoS One 2017 Dec;12(12):e0189393. https://doi. org/10.1371/journal.pone.0189393. PubMed (https://www. ncbi.nlm.nih.gov/entrez/query.fcgi?cmd=Retrieve\&db=PubM ed\&list_uids=29281675\&dopt=Abstract)

12. Ogden NH, Maarouf $A$, Barker IK, Bigras-Poulin M, Lindsay LR, Morshed MG, O'callaghan CJ, Ramay F, Waltner-Toews $D$, Charron DF. Climate change and the potential for range expansion of the Lyme disease vector Ixodes scapularis in Canada. Int J Parasitol 2006 Jan;36(1):63-70. https://doi. org/10.1016/j.ijpara.2005.08.016. PubMed (https://www. ncbi.nlm.nih.gov/entrez/query.fcgi?cmd=Retrieve $\& d b=P u b M$ ed\&list_uids=16229849\&dopt=Abstract)

13. Leighton PA, Koffi JK, Pelcat Y, Lindsay LR, Ogden NH. Predicting the speed of tick invasion: an empirical model of range expansion for the Lyme disease vector Ixodes scapularis in Canada. J Appl Ecol 2012;49(2):457-64. https:// doi.org/10.1111/j.1365-2664.2012.02112.x.

14. Statistique Canada. Estimations de la population au 1er juillet, par âge et sexe. Ottawa (ON): Government of Canada; 2017. https://www150.statcan.gc.ca/t1/tbl1/fr/tv.act ion?pid=1710000501\&request_locale=fr

15. Wadkins L. The forest resources of Ontario 2011. Sault Ste. Marie (ON): Ontario Ministry of Natural Resources, Forest Evaluation and Standards Section, Forests Branch; 2011.

16. Ministry of Health and Long-Term Care. Appendix B: Provincial case definitions for reportable diseases: Lyme disease. Toronto (ON): Government of Ontario; 2017. http:// 
www.health.gov.on.ca/en/pro/programs/publichealth/oph_ standards/docs/lyme_disease_cd.pdf

17. Ontario Agency for Health Protection and Promotion (Public Health Ontario). Ontario Lyme disease map 2017: estimated risk areas map. Toronto (ON): Queen's Printer for Ontario; 2017 Jun. https://www.publichealthontario.ca/en/ eRepository/Lyme_disease_risk_areas_map_2017.pdf

18. Nelder MP, Russell C, Lindsay LR, Dhar B, Patel SN, Johnson S, Moore S, Kristjanson E, Li Y, Ralevski F. Population-based passive tick surveillance and detection of expanding foci of blacklegged ticks Ixodes scapularis and the Lyme disease agent Borrelia burgdorferi in Ontario, Canada. PLoS One 2014 Aug;9(8):e105358. https://doi.org/10.1371/ journal.pone.0105358. PubMed (https://www.ncbi.nlm. nih.gov/entrez/query.fcgi?cmd=Retrieve\&db=PubMed\&li st_uids=25171252\&dopt=Abstract)

19. Nelson CA, Saha S, Kugeler KJ, Delorey MJ, Shankar MB, Hinckley AF, Mead PS. Incidence of clinician-diagnosed Lyme disease, United States, 2005-2010. Emerg Infect
Dis 2015;21(9):1625-31. https://dx.doi.org/10.3201/ eid2109.150417

20. Hinckley AF, Connally NP, Meek JI, Johnson BJ, Kemperman MM, Feldman KA, White JL, Mead PS. Lyme disease testing by large commercial laboratories in the United States. Clin Infect Dis 2014 Sep;59(5):676-81. https://doi.org/10.1093/ cid/ciu397

21. Falco RC, Fish D. Ticks parasitizing humans in a Lyme disease endemic area of southern New York State. Am J Epidemiol 1988 Nov;128(5):1146-52. https://doi.org/10.1093/ oxfordjournals.aje.a115057. PubMed (https://www.ncbi.nlm. nih.gov/entrez/query.fcgi?cmd=Retrieve $\& d b=$ PubMed\&li st_uids=3189288\&dopt=Abstract)

22. Stafford KC. Tick management handbook: an integrated guide for homeowners, pest control operators, and public health officials for the prevention of tick-associated disease, 2cd edition. The Connecticut Agricultural Experiment Station, Bulletin No. 1010, New Haven, CT (USA), 78 pp. https://stacks.cdc.gov/view/cdc/11444 